\title{
Pathobiology
}

Baranowski, W. 70

Bautista, P.A. 127, 140

Bombi, J.A. 79

Buda, I. 70

Bueno, G. 61

Capron, F. 148

Cierniak, S. 70

Deniz, O. 61

Evans, A.J. 121

Farahani, N. 121

Fernández-Carrobles, M.M. 61

Garcia, A. 79

García-Rojo, M. 57, 61, 99

Griffin, M. 140
Haneishi, H. 127

Hashimoto, N. 127

Herlin, P. 156

Kopik, J. 70

Kozłowski, W. 70

Laurinaviciene, A. 156

Laurinavicius, A. 156

Markiewicz, T. 70

Michaelson, J. 140

Mira, A. 89

Murawski, P. 70

Nap, M. 110
Ordi, J. 57, 79, 89

Pantanowitz, L. 121

Patel, K. 140

Patera, J. 70

Plancoulaine, B. 156

Racoceanu, D. 148

Rakislova, N. 89

Ramírez, J. 79, 89

Riben, M. 121

Saco, A. 79, 89

Senter-Zapata, M. 140

Słodkowska, J. 70

Snuderl, M. 127

Yagi, Y. 127, 140

\section{Subject Index Vol. 83, No. 2-3, 2016}

2D image reconstruction 127

3D reconstruction 140

- Slicer 140

Big data 148

Breast cancer 148

Cancer grading 148

- pathology report 70

Cost management 110

Data storage 110

- traffic 110

Digital image analysis 156

- pathology $61,99,110,121,148,156$

High-content image exploration 148

High-resolution 3D histology 140

Histology image 127
Image analysis 61

Image 140

Immunohistochemistry 156

Informatics 121

Integrative digital pathology 148

International guidelines 99

- telepathology 121

Medical education 79

Micro-CT 140

Ovarian cancer 70

Planning 110

Primary diagnosis 89

Proteomics 156
Routine diagnosis 89

Semantics 148

Standards 61

Structured synoptic report 70

Teaching 79

Technical specification 99

Tumor heterogeneity 156

\section{Validation 89}

Virtual microscopy $61,79,89,148$

Volume measurements 140

Whole organ 127

Whole-slide image(s) 89, 99

- imaging 61, 79, 121 